\title{
El buen vivir y la inclusión: Una reflexión desde la legislación educativa ecuatoriana
}

\author{
Good living and Inclusion: A Reflection From the Ecuadorian Educational Legislation
}

Buen Vivir e inclusão: uma reflexão da lei educacional equatoriana

Christian Xavier Chamorro-Pinchao

Universidad Nacional de Educación

Azogues, Ecuador

xavocha@hotmail.com

iD http://orcid.org/0000-0003-0988-3121

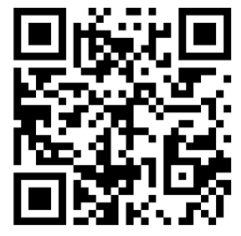

Genesis Noemi Loor-Cedeño

Universidad Nacional de Educación

Azogues, Ecuador

genesis.loor-unae@unaeedu.onmicrosoft.com

(iD) http://orcid.org/0000-0001-7307-3209

Julia Stefanía Agreda-Gómez

Universidad Nacional de Educación

Azogues, Ecuador

stephany.agre27@hotmail.com

iD http://orcid.org/0000-0003-4794-0289

Recibido • Received • Recebido: 10 / 08 / 2019

Corregido • Revised • Revisado: 11 / 02 / 2021

Aceptado • Accepted • Aprovado: 05 / 04 / 2021

Resumen: El presente artículo pretende reflexionar sobre la concordancia entre el las concepciones del buen vivir, la inclusión y la legislación educativa ecuatoriana, en los planteamientos generales del currículo 2016. La relevancia del trabajo radica en que el currículo ecuatoriano del 2016 guía la formación de las personas que habitan en la sociedad, en razón de lo cual es importante analizar su alineación con el buen vivir, como paradigma de desarrollo constitucional y con los principios de la inclusión, como garante de equidad social. Como proceso metodológico, se realiza una revisión documental que contempla el periodo 2008-2016, expuesta en tres epígrafes: Aproximación al buen vivir desde el Ecuador, fundamentos de la inclusión educativa, y revisión del currículo ecuatoriano desde el buen vivir y la inclusión. Como resultados surge que, en el componente armonía interna, el currículo ecuatoriano aborda, desde lo didáctico y metodológico, el desarrollo del estudiantado como un ser integral; en el componente armonía social no se conceptualiza, dentro del currículo, el respeto de una manera concreta y como bien básico del ser humano; y, el componente de armonía con la naturaleza no se apoya en acciones concretas que permitan su desarrollo dentro del currículo. 
http://doi.org/10.15359/ree.25-2.12

http://www.una.ac.cr/educare

educare@una.ac.cr

Se concluye que el currículo ecuatoriano refleja esa pretensión de transformar la sociedad; no obstante, no se proponen acciones concretas para alcanzar una educación inclusiva, concordante con las dimensiones analizadas del buen vivir. Por lo tanto, es necesario un plan de acciones viables que aporten al proceso de construcción de una educación inclusiva, concordante con el buen vivir.

Palabras claves: Buen vivir; currículo; Ecuador; educación; inclusión; legislación.

Abstract: This article aims to reflect on the concordance between the conceptions of good living, inclusion, and the Ecuadorian educational legislation in the general approaches of the 2016 curriculum. The relevance of the work lies in the fact that the 2016 Ecuadorian curriculum guides the formation of people living in society, so it is important to analyze the curriculum alignment with good living as a paradigm of constitutional development and with the principles of inclusion, as a guarantor of social equity. As a methodological process, a documentary review is conducted to analyze the period 2008-2016. The review was organized in three components as follows: an approximation to good living from Ecuador, fundamentals of inclusive education, and a revision of the Ecuadorian curriculum from good living and inclusion. The results show that, in the internal harmony component, the Ecuadorian curriculum considers the development of students as integral beings from the didactic and methodological point of view. In the social harmony component, respect is not conceptualized in a concrete way in the curriculum and as a basic good of the human being. Finally, the harmony with nature component is not supported by concrete actions allowing it to be developed within the curriculum. It is concluded that the Ecuadorian curriculum reflects the purpose of transforming the society; however, no concrete actions are proposed to achieve inclusive education, consistent with the analyzed dimensions of good living. Therefore, a viable action plan is needed to contribute to the process of building an inclusive education in accordance with good living.

Keywords: Buen vivir; curriculum; Ecuador; education, inclusion, legislation.

Resumo: Este artigo tem como objetivo refletir sobre o acordo entre Buen Vivir, inclusão e legislação educacional equatoriana, nas propostas gerais do currículo 2016. A relevância do trabalho reside no fato de que o currículo equatoriano 2016 orienta a formação de pessoas que vivem em sociedade. Portanto, é importante analisar seu alinhamento com o Buen Vivir, como paradigma de desenvolvimento constitucional e com os princípios da inclusão, como garante da equidade social. Como processo metodológico, é realizada uma revisão documental, abrangendo o período 2008-2016, exposta em três seções: Abordagem ao Buen Vivir do Equador, Fundamentos da inclusão educacional e Revisão do currículo equatoriano desde o Buen Vivir e inclusão. Como resultado, parece que: no componente Armonía interna, o currículo equatoriano aborda o desenvolvimento do corpo discente como integrante do ponto de vista didático e metodológico; na componente Armonía social, o respeito não é conceituado de forma concreta e como um bem humano básico no currículo; e, o componente de Armonía con la naturaleza não depende de ações concretas que permitam seu desenvolvimento no currículo. Conclui-se que o currículo equatoriano reflete essa pretensão de transformar a sociedade, porém, não são propostas ações concretas para o alcance de uma educação inclusiva, consistente com as dimensões do Buen Vivir analisadas. Portanto, é necessário ter um plano de ação viável que contribua para o processo de construção de uma educação inclusiva e coerente com o Buen Vivir.

Palavras-chave: Buen vivir; currículo; Equador; educação; inclusão; legislação. 


\section{Introducción}

Grandes avances tecnológico-científicos, así como crisis económicas, políticas y sociales forman parte del legado más significativo que ha dejado el pasado siglo XX, en la memoria y sentir colectivo. Sistemas de gobierno contradictorios han llevado las riendas de una sociedad mundial vulnerable, desinformada y dividida. Durante buena parte de la segunda mitad del siglo XX, corrientes políticas conservadoras han promulgado un modo de desarrollo basado en la competitividad desigual, en la privatización de los servicios públicos fundamentales y en la concentración del poder en pequeños grupos elitistas. Es así como Pérez-Morón y Cardoso-Ruiz (2014) mencionan que el proceso global de desarrollo ha implicado efectos contrapuestos al sentido de igualdad en la repartición de la riqueza y, de otro modo, ha beneficiado a grupos de poder que monopolizan las relaciones de producción, promulgan las relaciones mercantilistas de consumo y aprueban la explotación de los recursos naturales, para satisfacción de intereses económicos particulares.

Por tanto, dentro del modelo neoliberal, la prioridad no fue precisamente el desarrollo integral de la sociedad en su conjunto, sino, más bien, el enriquecimiento de aquellos grupos poseedores del capital; la explotación y empobrecimiento de quienes no contaban con los recursos para entrar, de manera igualitaria, en la contienda productiva. En este sentido, América Latina se vio afectada, al igual que el resto del mundo, por una sucesión de gobiernos entregados al sistema neoliberal predominante en la época, lo cual llevó al colapso socio económico de la región (Katz, 2014).

Particularmente en Ecuador, las políticas públicas estuvieron encauzadas en replicar y consolidar el sistema neoliberal, en donde las expectativas de progreso se encontraban en los resultados de las empresas privadas y en su capacidad de manejo de los recursos colectivos. En sentido particular, el enfoque de las políticas públicas en educación, promulgadas por el Estado, respondían a estos intereses financieros particulares, desde su administración, organización y finalidad (Acosta, 2005). Por tanto, se puede entender que la inversión en educación no representó una prioridad dentro de los intereses del Estado, como tampoco lo fue el otorgarle un sentido de construcción social, permanente y de calidad.

De otro modo, en el año 2008 se aprobó, mediante consulta popular, una nueva Constitución en el Ecuador, la cual busca promover los derechos de las personas sobre los intereses del sistema público y privado, regido por una lógica mercantilista (Secretaria Nacional de Planificación y Desarrollo [SENPLADES], 2014). De esta manera, la nueva carta magna fijó la visión de sociedad que el Estado ecuatoriano buscaba alcanzar. En esta línea, el gobierno diseñó el Plan Nacional para el Buen Vivir (PNBV), que encarna los principios ideológico políticos de esa nueva sociedad (León Guzmán, 2015). De acuerdo con el nuevo modelo social, el buen vivir, el criterio empleado para la distribución de los recursos se enfoca en el desarrollo de las 
http://doi.org/10.15359/ree.25-2.12

http://www.una.ac.cr/educare

educare@una.ac.cr

potencialidades y capacidades de la población, como reflejo de la garantía de sus derechos (SENPLADES, 2014). Es decir, la redistribución de los recursos públicos prioriza la formación y capacitación de la ciudadanía, a través de programas enfocados al fortalecimiento de la matriz productiva y la promoción de un país más competitivo, desde su talento humano.

En relación con lo expuesto, el presente artículo pretende reflexionar sobre la concordancia entre los principios del buen vivir y de la inclusión en la educación ecuatoriana. La relevancia del tema radica en que la legislación educativa analizada, el currículo 2016, debería ser consecuente con el paradigma de desarrollo, el buen vivir en el caso ecuatoriano, para contribuir a las metas planteadas por el país; así también, en que la articulación o no de los principios de inclusión con la legislación educativa brinda luces sobre la construcción de un piso ideológico y legal que viabilice una educación para todas las personas.

El desarrollo de este trabajo se enmarca en el periodo comprendido entre el año 2008 al 2016. Esto responde a tres hechos que marcan un hito: 1. en el 2008 se aprueba la vigente Constitución ecuatoriana; 2. alineado con los principios enunciados en la carta magna se dispone la creación del Plan Nacional del Buen Vivir en el año 2013, documento que redirecciona el sentido de la política pública; 3. en el 2016 se actualiza el currículo vigente para la educación obligatoria ecuatoriana desde los elementos enunciados tanto en la Constitución como en el Plan Nacional del Buen Vivir. En este sentido, el texto se ha estructurado de la siguiente manera: en el primer apartado presenta una aproximación al buen vivir desde el enfoque ecuatoriano, partiendo de sus antecedentes hasta la Constitución del 2008; el segundo apartado aborda los fundamentos políticos y educativos de la inclusión, y el tercer apartado realiza una revisión de los aspectos generales del currículo ecuatoriano, desde las dimensiones del buen vivir y la inclusión.

\section{Aproximación al buen vivir desde el Ecuador}

Tras una larga aplicación de recetas externas que evidenciaron un sistema decadente y cada vez menos compatible con las verdaderas necesidades de los pueblos latinoamericanos, una serie de movimientos sociales, ideológicos y políticos inician un proceso de ruptura con las visiones conservadoras (Arteaga-Cruz, 2017). Ecuador, en el año 2008, puso en marcha una nueva Constitución que cuenta con reformas estructurales que priorizan el desarrollo del individuo antes que al capital. De acuerdo con la SENPLADES (2014), "la nueva Constitución otorga derechos completos (incluyendo los sociales y económicos) a todos los ciudadanos y, además, obliga al Estado a priorizar el pago de la deuda social por encima de cualquier otra obligación" (p. 14). Este hecho se presentó como un referente a nivel regional e internacional, lo cual obedece a una nueva forma de gobierno que inició con el mandato del presidente Rafael Correa, a partir del 15 de enero del año 2007. 
http://doi.org/10.15359/ree.25-2.12

De acuerdo con Martínez Dalmau (2009), la Constitución ecuatoriana del año 2008 respalda y evidencia el cambio que los países latinoamericanos reclaman tras una memoria triste, inequitativa y desalentadora. De ahí que, según SENPLADES (2014), "el Sur no puede continuar siendo cómplice de un modelo de vida que hoy ha fracasado en el norte. El neoliberalismo ya fue experimentado en varios de nuestros países $y$, después de sus terribles golpes, aprendimos la lección" (p. 12). Por tanto, el valor social de la nueva Constitución ecuatoriana va más allá de un proyecto político particular, debido a que fungió como el nuevo horizonte que permitirá desestructurar un modelo hegemónico y clasista para transformarlo en uno que brinde oportunidades, en igualdad de condiciones, como alternativa para el desarrollo individual y colectivo de la sociedad.

Tal como lo mencionan Acevedo Rodríguez y Valenti Negrini (2017), "se ha impulsado en Ecuador un marco normativo que tiene como eje central promover la inclusión social, y se ha generado una importante estructura organizativa para diseñar e implementar programas sociales en este sentido" (pp. 153-154). En efecto, la normativa constitucional redirecciona el sentido antagónico que tenía la noción de desarrollo en la época neoliberal y lo relaciona con un sentido de convivencia armónica, de respeto a la diversidad y de promulgación del buen vivir o Sumak Kawsay; que según Álvarez González (2016) puede ser considerado como un principio ontológico, epistemológico, pedagógico y político de la sociedad.

En la Constitución del Ecuador, dentro de sus principios fundamentales, se menciona que "Art. 1.- el Ecuador es un Estado constitucional de derechos y justicia, social, democrático, soberano, independiente, unitario, intercultural, plurinacional y laico" (Asamblea Nacional Constituyente del Ecuador, 2008, p. 8). En este sentido, el buen vivir se presenta como el mecanismo que permite garantizar el goce y cumplimiento de los derechos de los individuos tomando en cuenta la diversidad y una forma ancestral propia de entender el mundo. El denominado Buen Vivir o Sumak Kawsay refleja una alternativa, de los pueblos originarios, a la perspectiva de desarrollo occidental, que plantea el elemento económico como eje central de interés. Es decir, el buen vivir, como paradigma de desarrollo, trasciende la pugna del poder económico, presente en la vía hacia el desarrollo, e integra aspectos diversos de la riqueza social, cultural y natural, en la centralidad del debate (Pérez-Morón y Cardoso-Ruiz, 2014).

El buen vivir o sumak kawsay es una filosofía de vida tomada de la cosmovisión andina que, a partir de una democracia participativa, pretende alcanzar el bienestar colectivo mediante la práctica de principios igualitarios, comunitarios y de reciprocidad ciudadana y con la naturaleza (León Guzmán, 2015; Ortiz, 2009). Esta noción para el desarrollo busca encaminar a la sociedad hacia una cultura de paz, de respeto e interdependencia para lograr objetivos comunes que permitan alcanzar, como fin principal, la felicidad. De tal manera, no es precisamente una novedosa propuesta de un partido o corriente política; por el contrario, condensa una cosmovisión ancestral de los pueblos originarios, en la que el sentido de la vida cobra un rumbo distinto y se 
http://doi.org/10.15359/ree.25-2.12

http://www.una.ac.cr/educare

educare@una.ac.cr

entrelaza con el bienestar del otro ser, como principio para el bienestar propio Es una alternativa de organización social que prioriza los derechos culturales, sociales y políticos de los individuos que componen las sociedades, sin desvincularse de los demás seres vivos existentes.

Para cristalizar el paradigma del buen vivir dentro de un proyecto nacional, el gobierno ecuatoriano, a través de la SENPLADES, crea el Plan Nacional del Buen Vivir 2013-2017, en el cual se postulan las prioridades del Estado a partir de grandes ejes estructurales. De acuerdo con la SENPLADES (2014), "la aplicación del Plan para el Buen Vivir implica un proceso de rupturas de distinta naturaleza, que constituye el motor que genera la transformación en el país, y que representa un resultado de coherencia con los principios programáticos del Gobierno" (p. 16). Con base en lo anterior, reestructurar la realidad de un país, mediante la implementación de un nuevo paradigma de desarrollo, requiere, por parte de las instituciones del Estado, un gran esfuerzo, organización, voluntad y compromiso por el cambio; pero, fundamentalmente, de una autonomía de los intereses de los grupos de poder, que les permita elaborar y tomar decisiones en cuanto a las políticas públicas requeridas.

En esta línea, la aplicación del buen vivir, en un nuevo proyecto de país, requiere del fortalecimiento de la relación entre la sociedad y el Estado, así como del fortalecimiento del rol central de este último como mediador y promulgador de la construcción social armónica y cooperativa. En palabras de Álvarez González (2016): "el Buen Vivir implica una educación por la convivencia, el reconocimiento y la inter-relación con la vida en todas sus expresiones: individuales, comunitarias, grupales, autárquicas, anárquicas, solidarias, diversas, naturales y culturales" (p. 7). Partiendo de este paradigma como eje rector de la reconstrucción de la sociedad ecuatoriana, es imprescindible promover, dentro del sentido social, la no exclusión a lo diferente, a lo desconocido, a todo aquello que provoca miedo o desconcierto y, por el contrario, adherir las potencialidades y capacidades diversas que aportan los distintos colectivos y grupos sociales, dentro de la composición de un Estado soberano.

\section{Fundamentos de la inclusión educativa}

La educación ha experimentado procesos constantes de exclusión e inclusión, lo cual va en concordancia con los fenómenos sociales vivenciados en cada época (Ramírez Valbuena, 2017). En esta sintonía "la exclusión social se ha abordado desde dos perspectivas en América Latina: una centrada en activos y oportunidades y otra que enfatiza los derechos ciudadanos" (Acevedo Rodríguez y Valenti Negrini, 2017, p. 153). La primera perspectiva se refiere a que los seres humanos que cuenten con una menor cantidad de recursos intelectuales, físicos y sociales tienen limitadas posibilidades de acceder a estructuras de oportunidades que les puedan brindar mejores condiciones de vida. Mientras que la segunda perspectiva trata el tema de los derechos ciudadanos, y menciona que los individuos excluidos no son partícipes de la legislación de las políticas públicas. 
http://doi.org/10.15359/ree.25-2.12

Desde esta perspectiva, cabe mencionar que se ha buscado luchar contra la exclusión desde diferentes ámbitos, principalmente, políticos y educativos, en busca de crear sociedades más equitativas, que brinden mejores condiciones de vida a la población. Un importante referente es la conferencia de Tailandia Educación para Todos, en donde, desde una perspectiva amplia, se aborda la necesidad de mejorar la adecuación de la educación, fortalecer su calidad y promover su accesibilidad universal, partiendo de las considerables deficiencias evidenciadas en el sistema educativo (Organización de las Naciones Unidas para la Educción, la Ciencia y la Cultura [UNESCO], 1990). De esta manera, se valora la necesidad de renovar la educación básica para que tenga un alcance amplio, reconociéndose el fracaso de los anteriores compromisos internacionales; y aproximándose, cada vez más, a la noción de inclusión.

Otro avance importante fue la conferencia de Salamanca, donde se establece que "la integración de niños con discapacidades deberá formar parte de los planes nacionales de 'Educación para Todos'" Organización de las Naciones Unidas para la Educación, la Ciencia y la Cultura, UNESCO, 1994, p. 18). En esta conferencia se realiza un énfasis en la educación como un derecho para todas las personas, con la visión de integrar a las personas con discapacidad dentro del sistema educativo regular. Por tanto, se rechaza, de manera enérgica, las instituciones que acentúan la exclusión de ciertos alumnado por determinadas capacidades físicas o intelectuales.

Siguiendo esta línea, se constata que los primeros manifiestos contra la exclusión se dan en un ámbito socio político, debido a que "la inclusión no es un concepto de exclusividad de la educación, su génesis radica en pautas de interacción social establecidas en cualquier contexto en el que el instinto gregario del ser humano se halle a flor de piel" (Rodríguez Corredor, 2013, p. 405). Esta afirmación muestra que las primeras intenciones de inclusión son plasmadas en compromisos basados en la justicia y equidad social, congruentes con los derechos de las personas con discapacidad. Es decir, se plantea una conciencia social que facilite, a los seres excluidos, el acceso a las herramientas necesarias para el goce pleno de sus derechos y, de la misma forma, que les permita ejecutar acciones determinadas que reduzcan su condición.

En este sentido, se replantea la discapacidad:

Analizándola como un fenómeno multidimensional, dinámico y cambiante que no se ubica en el cuerpo de individuo sino en la relación individuo-entorno; siendo el contexto donde se desenvuelve el individuo el que genera y valida la identidad discapacitante del sujeto y la lectura que los otros individuos pertenecientes al contexto le dan a esta condición. (Rodríguez Corredor, 2013, p. 405)

A partir de este aporte, se debe remarcar la necesidad de posicionar la lucha contra la exclusión del ser diferente como un tema de interés social, debido a que es en los espacios sociales donde se señalan qué cuerpos son los capacitados y cuáles los discapacitados. Por lo tanto, la inclusión se desarrolla cuando se da una experiencia compartida socialmente amplia, 
http://doi.org/10.15359/ree.25-2.12

http://www.una.ac.cr/educare

educare@una.ac.cr

con la participación activa de todas las personas, en busca de la igualdad de posibilidades y oportunidades individuales, que contribuyan al bienestar colectivo (Sen, 2001 citado por la UNESCO, 2008). De acuerdo con lo citado, se plantea la inclusión como un modelo de sociedad, "un proceso continuo uno de cuyos componentes es la educación y, para algunos incluso, la 'piedra angular"' (UNESCO, 2008, p. 7). Es así como, en cada sociedad, se crea una concepción de inclusión educativa que debe poner al alcance de los ciudadanos y las ciudadanas las oportunidades potenciadoras del desarrollo, atendiendo la diversidad humana, eliminando la desigualdad y la exclusión.

Cabe mencionar que la educación inclusiva se fundamenta en que educarse es un derecho fundamental y la vía para crear equidad y justicia social. Para lograr este cometido es fundamental que el Estado garantice que la ciudadanía no sea marginada o excluida del sistema educativo, que reciba una educación de calidad y que el tema de la cobertura no sea un impedimento real para que se logren cubrir las metas propuestas. En caso de que la educación siga siendo restringida a unos sectores de la población, la ciudadanía con menor preparación académica terminará cubriendo labores o tareas de baja remuneración y calificación, lo cual devendría en un aumento de los índices de pobreza (de la Cruz Flores, 2017). Desde esta perspectiva se debe considerar que se mejoraran las condiciones de la población, en la medida en que la educación sea adaptada para recibir a todas las personas. En este panorama, la cobertura y calidad son factores complementarios de una inclusión educativa que debe "reconocer el valor intrínseco de la diversidad y el respeto de la dignidad humana" (UNESCO, 2015 citada en UNESCO, 2017, p.18).

A partir de esta revisión, se puede afirmar que el Ecuador debe crear las condiciones sociales para favorecer al pleno desarrollo de las capacidades y potencialidades de todos y todas, para estar articulado con los principios declarados en su paradigma de desarrollo, el buen vivir, que busca una equidad y justicia social. Dentro de este ámbito, la educación constituye un eje vertebral debido a que la inclusión de los sectores históricamente excluidos, en el ámbito académico científico, brinda herramientas de lucha contra las desigualdades sociales. En este sentido, la inclusión educativa debe ser estipulada desde el proyecto educativo nacional (UNESCO, 2008).

En el caso de Ecuador, el Ministerio de Educación (2011) propone, como ejes fundamentales, el respeto a la diversidad y una buena calidad educativa que dé a cada persona lo que necesita, y no precisamente, dar a todos y a todas lo mismo y al mismo tiempo. Es decir, la calidad educativa en el Ecuador busca responder a las necesidades específicas de los sujetos educativos, tomando en cuenta su situación real de vulnerabilidad sin generalizar los posibles requerimientos que tenga cada individuo. Así mismo, la educación inclusiva ecuatoriana reconoce a cada persona como un ser único, puesto que, cada estudiante tiene su propio estilo y ritmo de aprendizaje; necesidades educativas, intereses, motivaciones y capacidades diferentes al resto de sus compañeros y compañeras, por motivo de sus diferentes orígenes de culturales, sociales y sus características personales (Ministerio de Educación, 2011). 
http://doi.org/10.15359/ree.25-2.12

\section{Revisión del currículo ecuatoriano desde el buen vivir y la inclusión}

En el art. 341 de la constitución ecuatoriana del 2008 se establece que el Estado generará:

Las condiciones para la protección integral de sus habitantes a lo largo de sus vidas ... en particular la igualdad en la diversidad y la no discriminación, y priorizará su acción hacia aquellos grupos que requieren consideración especial por la persistencia de desigualdades, exclusión, discriminación o violencia, o en virtud de su condición etaria, de salud o de discapacidad. (Asamblea Nacional Constituyente del Ecuador, 2008, p. 106)

En este sentido, el Estado debe garantizar la igualdad de oportunidades para todos los ciudadanos y todas las ciudadanas mediante la promoción de políticas de inclusión en los diferentes ámbitos de la sociedad, tales como la educación. La carta magna menciona que la educación es un derecho para todas las personas, la cual debe centrarse en el desarrollo justo e igualitario del ser humano. De este modo, representa una prioridad el garantizar su libre acceso, sin ningún tipo de restricción o discriminación educativa. Por lo tanto, se fomenta la libertad, equidad e igualdad de enseñanza, de manera participativa, incluyente, democrática y de calidad.

A partir del año 2008, el sistema educativo del Ecuador ha implementado el desarrollo de la educación inclusiva con el fin de fomentar una Educación para Todos (Ministerio de Educación, 2011). De manera que se han realizado algunos cambios en los aspectos curriculares, los cuales, brindan nociones de actuación al interior de la comunidad educativa, con la finalidad de responder a las necesidades de cada estudiante. A partir de lo anterior, se puede afirmar que el sistema educativo busca el bienestar de los educandos, además de brindar un proceso formativo de calidad y calidez.

En relación con el currículo, Gimeno Sacristán (2012) menciona que es un plan de estudio que guía tanto al personal docente como a los estudiantes y las estudiantes. Así mismo, en el currículo se especifican los contenidos a enseñar y aprender y se regula la enseñanza durante la vida académica. De este modo, toma en cuenta aspectos como diferencias de género, culturales, religiosas, lingüísticas, étnicas, afectivas, motrices y cognitivas; de tal forma que se pueda ofrecer un currículo sin exclusiones y muy amplio. En este mismo sentido, López (2004) menciona que en la creación del currículo común no se pretende proponer una pedagogía homogénea; por el contrario, se busca romper con lo planificado debido a que origina desigualdades. Considerando esta postura, se debe resaltar la importancia de que el personal docente diseñe propuestas de enseñanza aprendizaje, tomando en cuenta la experiencia y el contexto en el que se desarrolla el estudiantado.

En esta lógica, el Ministerio de Educación (2016) prescribe al currículo como un plan estructural que se basa en fomentar una convivencia justa y equitativa, a partir de la inclusión social. Es decir, trata de crear e incentivar espacios de inclusión estimulando en el estudiantado 
http://doi.org/10.15359/ree.25-2.12

http://www.una.ac.cr/educare

educare@una.ac.cr

un desarrollo integral y armónico. También, pretende que el alumnado aprenda de acuerdo a su ritmo y estilo de aprendizaje, contexto cultural y lengua, teniendo las mismas oportunidades y necesidades de querer trabajar en equipo.

Complementando lo citado, dentro de los planteamientos del Plan Nacional del Buen Vivir se expone el sentido que debe tener la educación para alcanzar los objetivos como país, en cumplimiento con la normativa constitucional. De tal forma, según la SENPLADES (2014), las rupturas al modelo excluyente surgen a partir de una revolución cultural, la cual pretende formar ciudadanos y ciudadanas con pensamiento crítico, comprometidos con los requerimientos, necesidades e intereses de los demás individuos.

Llegado a este punto, León Guzmán (2015) menciona que existen tres componentes neurálgicos del buen vivir, los cuales fungen como categorías de análisis para el abordaje de la inclusión en el currículo ecuatoriano. El primero, la armonía interna de las personas hace referencia al aspecto individual, equilibrio personal, ya sea espiritual, mental o físico. El segundo, la armonía social con la comunidad trata aspectos colectivos en donde se desarrollan propuestas comunes afianzadas en el respeto, equidad social y trabajo colaborativo. El tercero, la armonía con la naturaleza pretende afianzar el sentido de interdependencia con todos los seres vivos, no únicamente con los humanos, además de la promulgación de la sustentabilidad ambiental y el biocentrismo.

A partir de este desarrollo conceptual es pertinente analizar los tres componentes del buen vivir con los postulados generales de la educación obligatoria establecidos en la parte introductoria del currículo ecuatoriano 2016.

\section{- Armonía interna de las personas}

En lo que respecta a este componente, el Ministerio de Educación del Ecuador (2016) en los principios para el desarrollo del currículo, establece:

El presente currículo ha sido diseñado mediante destrezas con criterios de desempeño que apuntan a que los estudiantes movilicen e integren los conocimientos, habilidades y actitudes propuestos en ellas en situaciones concretas, aplicando operaciones mentales complejas, con sustento en esquemas de conocimiento, con la finalidad de que sean capaces de realizar acciones adaptadas a esa situación y que, a su vez, puedan ser transferidas a acciones similares en contextos diversos. (p. 13)

Es decir, el currículo ecuatoriano busca el desarrollo de destrezas con criterio de desempeño que integren los conocimientos, habilidades y actitudes de forma contextualizada, para que el estudiantado adquiera herramientas que le permitan enfrentar la diversidad, 
http://doi.org/10.15359/ree.25-2.12

lo cual implica que "la verdadera educación supone la formación consciente y práctica de nuevos hábitos, informados y contrastados" (Pérez Gómez y Pérez Granados, 2013, p. 77). Por tal motivo, la apuesta por fortalecer un pensamiento crítico que cuestione los estereotipos y estigmas sociales predeterminados debe representar, en el ejercicio práctico del currículo, un eje vertebrador y coherente del nuevo enfoque de la educación en Ecuador. Una educación que permita desarrollar en el individuo la imaginación, el pensamiento y el razonamiento es aquella que contribuye a alcanzar la armonía interna de las personas como uno de los componentes del buen vivir (Nussbaum, 2012).

Además, en el apartado de la multiplicidad de significados del concepto de aprendizajes básicos, se menciona:

Los aprendizajes que se pretende que todo el alumnado adquiera en el transcurso de la educación "básica" son o quieren ser, en esta acepción, una garantía para conseguir que, puedan proseguir sin problemas ni dificultades destacadas los procesos formativos posteriores ... o para que puedan continuar aprendiendo a lo largo de la vida. (Ministerio de Educación, 2016, pp. 26-27).

De acuerdo con lo planteado, se considera imprescindible que los aprendizajes básicos adquiridos por el estudiantado, en el transcurso de su formación, respondan a la emergencia de herramientas que garanticen un pleno desarrollo en la sociedad, y que, en consecuencia, contribuyan al desarrollo y valorización del sentido de inclusión y equidad, enmarcado en el debate educativo mundial. Es así como la UNESCO (2017) establece:

El Objetivo de Desarrollo Sostenible (ODS) 4 sobre la educación exige que se garantice una educación inclusiva y equitativa de calidad y se promuevan oportunidades de aprendizaje a lo largo de toda la vida para todas y todos de aquí a 2030. (p. 3)

Por otra parte, también se contribuye a la puesta en práctica de la creatividad, de la libertad de expresión y en sí, de un sentido propio de entender, experimentar y aprender de la cotidianidad de la vida, aportando a la armonía personal (León Guzmán, 2015). Siguiendo este orden de ideas, el Ministerio de Educación (2016) establece:

Los aprendizajes que se pretende que todo el alumnado adquiera en el transcurso de la educación "básica" son o quieren ser, en este significado del concepto, una garantía para promover la equidad, para compensar las desigualdades sociales y culturales y evitar que acontezcan desigualdades educativas, para impulsar la cohesión y la integración social. Son "básicos" los aprendizajes valorados como necesarios para todo el mundo, los aprendizajes que todos los niños, niñas y jóvenes tienen que conseguir para no quedar en situación de riesgo de segregación o de exclusión social. (p. 25) 
http://doi.org/10.15359/ree.25-2.12

http://www.una.ac.cr/educare

educare@una.ac.cr

A partir de lo anterior, se puede señalar que los aprendizajes que busca el currículo pretenden, principalmente, combatir la exclusión; en conformidad con lo manifestado por la UNESCO (2017), se quiere "movilizar a los principales actores claves del sistema educativo y de la comunidad para propiciar las condiciones para un aprendizaje inclusivo y una comprensión más amplia de los principios de inclusión y equidad" (p. 13), con lo cual se busca eliminar la marginación, y generar, así, armonía personal, "una garantía para promover una ciudadanía activa, constructiva, enriquecedora y satisfactoria tanto por las personas individuales como por la sociedad en general" (Ministerio de Educación, 2016, p. 26).

Todas estas observaciones se relacionan también con Stiglitz, Sen y Fitoussi (2010, citados por León Guzmán, 2015, p. 53), quienes mencionan que la educación "es importante en la provisión de destrezas y competencias que apuntalan la producción económica. Pero la educación importa para la calidad de vida independientemente de sus efectos sobre los ingresos y productividad de la gente". Aclaran que la educación debe brindar oportunidades para la inclusión económica, pero no es su principal fin, sino que se direcciona principalmente a que se puedan desarrollar herramientas para una vida de calidad. Por ello, el currículo pretende que, al finalizar la educación básica obligatoria, el estudiantado egresado "comprendemos las necesidades y potencialidades de nuestro país y nos involucramos en la construcción de una sociedad democrática, equitativa e inclusiva" (Ministerio de Educación, 2016, p. 8).

\section{- Armonía social con la comunidad y entre comunidades}

El currículo del Ecuador, en su apartado de orientaciones metodológicas, menciona:

Los principios para el desarrollo del currículo ... han de incidir en las programaciones didácticas que elaboren las instituciones educativas para los niveles de educación obligatoria, considerando la atención a la diversidad y el acceso de todo el alumnado a la educación como principios fundamentales de esta tarea. (Ministerio de Educación, 2016, p. 14)

De tal manera, se infiere que el currículo realiza un reconocimiento de las diferencias existentes en el estudiantado, tomando en cuenta que tratar diversidad, sin topar exclusión o inclusión, se vuelve imposible. Considerar y reconocer la diversidad implica pensar desde el respeto al ser otro, generando inclusión (Soto Builes, 2007). Por lo que, dentro de la educación, se busca fomentar, entre el estudiantado, buenas relaciones, un ambiente de armonía y comprensión, en donde todos y todas respeten las opiniones y actitudes del otro sr. Esto va de la mano con el Sumak Kawsay, debido a que no "es posible pensar en una educación para el Buen Vivir si no se reconoce y aprende de las resistencias centenarias, de las luchas de aquellos grupos, comunidades y pueblos y, de las alternativas educativas y pedagógicas gestadas por ellos" (Villagómez y Cunha de Campos, 2014, p. 38). Es importante resaltar que postular el reconocimiento a la diversidad y la necesidad de garantizar el acceso a todo el estudiantado establece la base que posibilita alcanzar una inclusión educativa, que concuerda con los principios del buen vivir. 
http://doi.org/10.15359/ree.25-2.12

Además, se postula como uno de los elementos del perfil de salida del bachillerato ecuatoriano" procedemos con respeto y responsabilidad con nosotros y con las demás personas, con la naturaleza y con el mundo de las ideas" (Ministerio de Educación, 2016, p. 8). Es decir, el currículo plantea que el estudiantado tiene que aprender a promover un clima de tolerancia y respeto, en todos los ámbitos. Persiguiendo este objetivo, el rol de las instituciones educativas se dirige a brindar atención a la diversidad del alumnado, desarrollando métodos y estrategias que respondan a los diferentes estilos y ritmos de aprendizaje existentes en el aula; y así, permitir el acceso al ámbito educativo a todos y todas. Sin embargo, aunque el currículo menciona el reconocimiento del otro ser, desde el respeto, no es definido como un bien básico inherente a la dignidad humana, requisito fundamental para una educación inclusiva (Furstenberg, 2015). Se alude brevemente a este punto, dentro de los objetivos del área de ciencia sociales, donde se menciona que la finalidad es "aplicar los conocimientos adquiridos, a través del ejercicio de una ética solidaria y ecológica que apunte a la construcción y consolidación de una sociedad nueva basada en el respeto a la dignidad humana y de todas las formas de vida" (Ministerio de Educación, 2016, p. 156). Se observa un vacío conceptual en los apartados generales del currículo, pues es necesario que no se relegue la conceptualización del respeto, como bien básico del ser humano, a un área determinada, sino que su noción sea transversal en la formación con pautas y estrategias claras que permitan alcanzar el objetivo del perfil del bachillerato ecuatoriano.

\section{- Armonía con la naturaleza}

En este sentido, el Ministerio de Educación (2016) en el perfil de salida del bachillerato ecuatoriano, específicamente, en el valor de justicia, menciona que el estudiantado al finalizar su educación obligatoria debe ser respetuoso y responsable con la naturaleza, igualmente, con las ideas diferentes que tienen el resto de las personas que lo rodean, tomar en cuenta el cumpliendo de cada una de sus obligaciones y realizar una observación exhaustiva en sus derechos. De tal manera que, mediante el proceso educativo los individuos deben adquirir las herramientas necesarias para convivir en armonía con todos los seres vivos existentes en la naturaleza. Sin embargo, esta capacidad que deben tener el estudiantado egresado de las instituciones educativas carece de medidas concretas que posibilitan su adecuado desarrollo, debido a que no se hace un hincapié en el área de la relación humano-naturaleza en los distintos apartados generales del currículo, considerado este como un aprendizaje básico.

Cabe mencionar que la superficialidad con la que es abordado el respeto a la naturaleza desde el currículo ecuatoriano no concuerda con las pretensiones del buen vivir en la educación. De acuerdo con lo que menciona León Guzmán (2015) la naturaleza representa una serie de elementos esenciales de la vida, con los que el ser humano interactúa permanentemente, a los cuales debe rendir respeto y responsabilidad; debido a que, desde una perspectiva indigenista, la naturaleza representa una dimensión sagrada. Así también, desde la perspectiva ecológica, se 
http://doi.org/10.15359/ree.25-2.12

http://www.una.ac.cr/educare

educare@una.ac.cr

promulga la sostenibilidad ambiental y el biocentrismo como nociones básicas que permiten el desarrollo colectivo. Por eso, la educación debe enfatizar el desarrollo del sentido de convivencia armónica con la naturaleza, a partir de acciones específicas que realmente conlleven a una transformación dentro del área educativa.

\section{A modo de conclusiones}

Tras el recorrido realizado durante esta reflexión, es pertinente abordar el siguiente cuestionamiento: ¿qué papel juega el buen vivir y la inclusión dentro de la legislación educativa ecuatoriana, específicamente en el currículo 2016? En esta línea, se ha evidenciado la institucionalización de principios filosóficos, sociales, económicos y políticos dentro de la legislación ecuatoriana en general. Particularmente, en la normativa de educación, la puesta en marcha del currículo 2016 pretende que los ciudadanos y las ciudadanas se desarrollen de forma integral, en concordancia con el buen vivir y con los principios de inclusión, plasmándolo en el perfil de salida del bachillerato ecuatoriano. No obstante, el ideal de ciudadanía planteado no se traduce en una propuesta sistemática y concreta, dentro de los ejes transversales, que posibilite la consecución de este objetivo.

Específicamente, el componente de armonía con la naturaleza, a pesar de ser aludido de manera breve en el apartado de perfil de salida del currículo, no se apoya en acciones específicas que permitan su desarrollo. De igual forma, respecto al componente armonía social con la comunidad y entre comunidades, en el perfil de salida del currículo se hace eco de una formación que respeta la diversidad en los diferentes ámbitos de la vida; sin embargo, no se conceptualiza el respeto de una manera concreta, como bien básico del ser humano, ni como un eje transversal en la formación del estudiantado. Por otra parte, en lo tocante al componente armonía interna de las personas, el currículo ecuatoriano aborda, desde lo didáctico y metodológico, como un aprendizaje básico esencial y como una de las metas del perfil de salida, el desarrollo del estudiantado como un ser integral.

Por tanto, es menester recomendar que el currículo ecuatoriano 2016 adopte culturas, políticas y prácticas inclusivas concretas que redireccionen la finalidad de la educación hacia la praxis del buen vivir y la inclusión, como ejes rectores del proceso de enseñanza aprendizaje. La transversalidad de estos ejes debe darse a lo largo de todo el proceso educativo, de forma sistemática y articulada, evitando responsabilizar a un área de conocimiento o a un nivel escolar especifico. La complejidad que demanda la cristalización, tanto de las dimensiones del buen vivir analizadas como de los principios de inclusión, requiere de la reconfiguración de los principios filosóficos, didácticos y pedagógicos del currículo, de manera que se viabilice un diálogo entre diferentes. 


\section{Declaración de Material complementario}

Este artículo tiene disponible, como material complementario:

-La versión preprint del artículo en https://doi.org/10.5281/zenodo.3765416

\section{Referencias}

Acevedo Rodríguez, C. y Valenti Nigrini, G. (2017). Exclusión social en Ecuador. Buen vivir y modernización capitalista. Polis, Revista Latinoamericana, 16(46), 151-174. https://doi. org/10.4067/S0718-65682017000100151

Acosta, A. (2005). Ecuador: Ecos de la rebelión de los forajidos. Nueva Sociedad, 198, 42-54. http://nuso.org/articulo/ecuador-ecos-de-la-rebelion-de-los-forajidos/

Álvarez González, F. J. (2016). ¿En qué puede devenir la educación a partir del paradigma del buen vivir? UNAE. http://201.159.222.12:8080/handle/56000/489

Arteaga-Cruz, E. L. (2017). Buen vivir (Sumak Kawsay): Definiciones, crítica e implicaciones en la planificación del desarrollo en Ecuador. Saúde em Debate, 41(114), 907-919. https://doi. org/10.1590/0103-1104201711419

Asamblea Nacional Constituyente del Ecuador. (2008). Constitución de la República del Ecuador. Montecristi, Ecuador.

de la Cruz Flores, G. (2017). Igualdad y equidad en educación: Retos para una América Latina en transición. Educación, 26(51), 159-178. https://doi.org/10.18800/educacion.201702.010

Furstenberg, C. (2015). La dignidad inherente al hombre a respetar, proteger y promover en todo ser humano: Una lucha que merece la pena para la protección de la humanidad. Horizonte de Enfermería, 26(2), 47-56. https://doi.org/10.7764/Horiz Enferm.26.2.47

Gimeno Sacristán, J. (2012). ¿Qué significa el currículum? En J. Gimeno Sacristán, R. Feito Alonso, P. Perrenoud y M. Clement Linuesa (Autores), Diseño, desarrollo e innovación del currículum (pp. 25- 47). Morata.

Katz, C. (2014). Neoliberales en América Latina. Ortodoxos y convencionales. Derecho y Realidad, 12(24), 233-256. https://doi.org/10.19053/16923936.v2.n24.2014.4546

León Guzmán, M. (2015). Del discurso a la medición: Propuesta metodológica para medir el buen vivir en Ecuador. INEC. http://www.ecuadorencifras.gob.ec/wp-content/uploads/ downloads/2016/10/Buen-Vivir-en-el-Ecuador.pdf 
http://doi.org/10.15359/ree.25-2.12

http://www.una.ac.cr/educare

educare@una.ac.cr

López, M. (2004). El sentido inclusivo del currículum. En M. López (Ed.), Construyendo una escuela sin exclusiones: Una forma de trabajar en el aula con proyectos de investigación (pp. 165211). Aljibe.

Martínez Dalmau, R. (2009). El proyecto de Constitución de Ecuador, ejemplo del nuevo constitucionalismo latinoamericano. IUS. Revista del Instituto de Ciencias Jurídicas de Puebla A.C., 23, 264-274. http://www.redalyc.org/articulo.oa?id=293222963011

Ministerio de Educación. (2011). Modulo I. Educación inclusiva y especial. https://educacion.gob. ec/wp-content/uploads/downloads/2013/07/Modulo Trabajo El.pdf

Ministerio de Educación. (2016). Currículo de los niveles de Educación Obligatoria. En Ministerio de Educación. https://educacion.gob.ec/wp-content/uploads/downloads/2016/03/ Curriculo1.pdf

Nussbaum, M. C. (2012). Crear capacidades. Crear capacidades. Propuesta para el desarrollo humano. Paidós.

Ortiz, P. (2009). Sumak kawsay en la constitución ecuatoriana de 2008: Apuntes en torno a sus alcances y desafíos. ALTERIDAD. Revista de Educación, 4(1), 76-87. https://doi.org/10.17163/ alt.v4n1.2009.06

Pérez Gómez, A. y Pérez Granados, L. (2013). Competencias docentes en la era digital. La formación del pensamiento práctico. Revista temas de educación, 19, 67-83. http://hdl. handle.net/10630/9702

Pérez-Morón, L. Y. y Cardoso-Ruiz, R. P. (2014). Construcción del buen vivir o Sumak Kawsay en Ecuador: Una alternativa al paradigma de desarrollo occidental. Contribuciones desde Coatepec, 26, 49-66. http://www.redalyc.org/articulo.oa?id=28131424004

Ramírez Valbuena, W. Á. (2017). La inclusión: Una historia de exclusión en el proceso de enseñanza aprendizaje. Cuadernos de Lingüística Hispánica, 30, 211-230. https://doi. org/10.19053/0121053X.n30.0.6195

Rodríguez Corredor, J. (2013, octubre). Del discurso de la inclusión a la invisibilización de las necesidades educativas especiales (NEE) en el aula. En Y. Pedraza Jimenez y O. Pulido Cortes (Eds.), Memorias Congreso Investigación y Pedagogía. III Nacional II Internacional. La educación en el siglo XXI: Ser, saber y producir en la incertidumbre y el caos. Universidad Pedagógica y Tecnológica de Colombia Tunja, Colombia.

Secretaría Nacional de Planificación y Desarrollo. (2014). Buen vivir. Plan Nacional 2013-2017. Todo el mundo mejor. http://www.fao.org/faolex/results/details/es/c/LEX-FAOC139396/ 
http://doi.org/10.15359/ree.25-2.12

Soto Builes, N. (2007). ¿Diversidad-inclusión vs transformación? El ágora usb, 7(2), 322-332. http://p.redalyc.org/articulo.oa?id=407748997011

Organización de las Naciones Unidas para la Educción, la Ciencia y la Cultura. (1990). Declaración mundial sobre educación para todos y marco de acción para satisfacer las necesidades básicas de aprendizaje. Autor.

Organización de las Naciones Unidas para la Educción, la Ciencia y la Cultura. (1994). Declaración de Salamanca y marco de acción sobre necesidades educativas especiales. https://www. educacionespecial.sep.gob.mx/pdf/doctos/3Internacionales/3DeclaracionSalamanca.pdf

Organización de las Naciones Unidas para la Educción, la Ciencia y la Cultura. (2008). La educación inclusiva: El camino hacia el futuro. http://www.ibe.unesco.org/fileadmin/user upload/ Policy Dialogue/48th_ICE/General_Presentation-48CIE-4 Spanish_pdf

Organización de las Naciones Unidas para la Educción, la Ciencia y la Cultura. (2017). Guía para asegurar la inclusión y la equidad en la educación. http://unesdoc.unesco.org/ images/0025/002595/259592s.pdf

Villagómez, M. S. y Cunha de Campos, R. (2014). Buen vivir y educación para la práctica de la interculturalidad en el Ecuador. Otras prácticas pedagógicas son necesarias. Alteridad. Revista de Educación, 9(1), 35-42. https://doi.org/10.17163/alt.v9n1.2014.03 